\title{
The Violent Universe
}

\author{
Nicola Omodei* \\ Stanford University \\ E-mail: nicola.omodeieslac.stanford.edu
}

In this paper I present some highlights from the "Astroparticle Physics" session, which I had the honor and the privilege to summarize in the plenary session. The excellent quality of the session and the large number of talks reflect the amount of new results obtained in the last year in this branch of physics. It was possible to include in my talk only a small fraction of what has been presented; I offer a personal, biased, and probably very limited view. It is surely not the only way to read and discuss the most recent years of research in this field so, take it as an introduction, and refer to the material presented in this proceedings, which I am sure will follow the excellent quality of the talks, to extract more precise and detailed information. Here, I focus on three main aspects that I found particularly relevant: the synergy between experiments, which to me is the key for turning an observation to a discovery, the issues that are still open, which could serve as inspiration for future work, and the prospects for the future.

35th International Conference of High Energy Physics - ICHEP2010,

July 22-28, 2010

Paris France

${ }^{*}$ Speaker. 


\section{A golden age for Particle Astrophysics}

Particle Astrophysics, or Astroparticle physics, is that branch of particle physics that studies elementary particles of astronomical origin, and their relation to astrophysics and cosmology. In the last few years we have been witnesses of a Golden Age for particle astrophysics, particularly characterized by successful joint ventures between particle physics, astronomy and cosmology. From one side, questions about the origin of the Cosmos and the content of the Universe have reached the particle physics community, asking, for example, questions about the extension of the Standard Model, and if this could give rise to a weakly interacting particle that constitutes the $23 \%$ of the mass-energy density of the Universe. On the other hand, the development of detectors for particle physics made accessible to astronomy a series of new technologies that led to the design of new experiments and infrastructures. Underground experiments, specially-designed telescopes, antennas, satellites, and world-wide distributed collaborations signing their papers in alphabetical order are the new labs for detecting a wide range of cosmic messengers.

\section{Dark Matter Searches}

Dark Matter (DM) is inferred to exist from the observation of missing mass in galaxy clusters, from the observation of flat rotation curves in galaxies, and from gravitational lensing. From the measurement of the anisotropies of the Cosmic Microwave Background, one can infer the basic properties of the Universe. In fact, measurements from WMAP [1] played a key role in establishing the current Standard Model of Cosmology, namely the $\Lambda$ CDM model, a flat universe dominated by dark energy, supplemented by dark matter and atoms with density fluctuations seeded by a Gaussian, adiabatic, nearly scale invariant process. One of the most favored candidates for a DM particle is the WIMP (Weakly Interacting Massive Particle). No convincing detection of a DM signal has been ever reported. Within the dark matter community, possible signals from the DAMA/LIBRA and CoGeNT experiments have aroused renewed interest in WIMPs. Direct searches are focused on measuring the WIMP-nucleon cross-section by means of atom recoil. The most important result was presented here by the CDMSII collaboration[2], who reported the combined results from CDMSII, a not statistically significant detection of 2 events in the signal region (when $0.9 \pm 0.2$ background events are expected). This led to the world-leading limit on spin-independent WIMP-

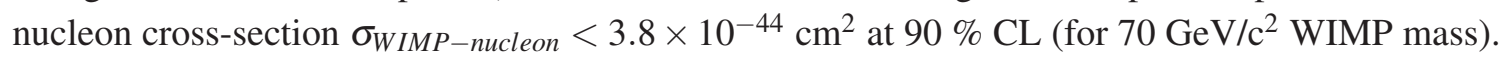
To reduce the contamination by surface events that mimic nuclear recoil in the signal region a series of upgrades and detector development has been proposed (new CDMS, but also see LUX[3]). Germanium detectors for low WIMP masses $(<10 \mathrm{GeV})$, with sub-keV recoil energies, have also been presented[4].

Celestial cosmic rays and gamma-rays are indirect probes for DM signals. In this case, the background for the detection of the signal is represented by astrophysical "foregrounds", which are often known with modest precision. In particular, regions where a strong DM signal is expected often have large uncertainties in their foreground (e.g., the center of our Galaxy is a particularly difficult region, due to, for example, a very likely unresolved population of point sources, an intense radiation field, and large quantities of gas along the line of sight). Also interesting are the results from a completely different strategy: searching for gamma-ray signals from regions where no 
signal from astrophysical sources is expected but where a high content of DM is expected. Dwarf spheroidal galaxies (dSph) are low luminosity galaxies, satellites of the Milky Way. Their massto-light ratios (derived from the dynamics of stellar motion) are particularly high and therefore dSphs are good candidates for finding gamma-ray signals from DM. Johnson, for the Fermi/LAT collaboration, and Glicenstein, for the H.E.S.S. collaboration[5, 6], have presented upper limits on $\langle\sigma v\rangle$ from the observations toward dSphs. The upper limits so obtained are starting to cut into the "interesting" phase space where some realizations of common models ${ }^{1}$ can be ruled out.

\section{Gamma-Ray Astrophysics}

Since its launch in June 2008, the Fermi Gamma-Ray Space Telescope has substantially contributed to the gamma-ray astrophysics with a series of new and important discoveries, representing a real revolution in this field ${ }^{2}$.

In this respect, I would like to highlight the the synergy between Fermi and on-ground detectors. Sources detected at $\mathrm{GeV}$ energies by Fermi[7] are studied at higher energies by Air Cherenkov Telescopes (ACT), such as H.E.S.S., MAGIC or VERITAS and Extensive Air Shower (EAS) detectors, like Milagro or ARGO-YBJ. In the case of ACT, the large collection area (the part of the upper atmosphere that is imaged) allows a good sensitivity to point sources, but the small field of view implies that a candidate list of sources becomes really helpful to target the observations. Regarding the overlap between astrophysics and particle physics, starburst galaxies are particularly interesting sources. Fermi, H.E.S.S., and VERITAS[8] have detected for the first time emission at $\mathrm{GeV}$ and $\mathrm{TeV}$ energies from M82 and NGC 253. The detected gamma ray emission is not associated with the presence of powerful jets, as for active galactic nuclei, but is rather related to the high star formation activity in the gas-rich central regions of the galaxies. In fact, massive stars, whose lives
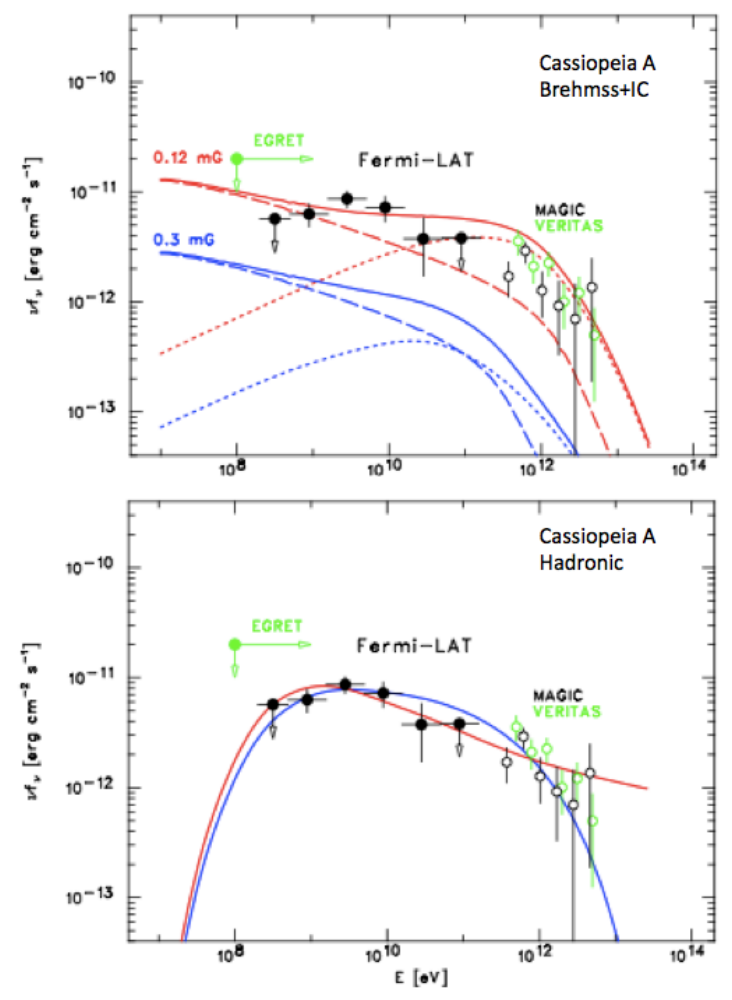

Figure 1: Joint observations between MAGIC, VERITAS, and Fermi/LAT of the SNR Cassiopeia A. Hadronic and leptonic models can be use to interpret the observed emission. are far shorter than those of low-mass stars, quickly undergo supernova explosions that efficiently accelerate particles in shocks of their remnants (SNRs). The detections show that the total gamma-ray fluxes of the sources is consistent

\footnotetext{
${ }^{1}$ The most constraining are related to Minimal Supersymmetric Standard Model (MSSM).

${ }^{2}$ For a complete list of published LAT team results: https://www-glast.stanford.edu/cgi-bin/pubpub
} 
with gamma-ray emission originating from the interaction of accelerated cosmic rays with local interstellar gas and radiation fields. This provides evidence for particles being accelerated in SNRs, and constitutes evidence for a link between massive star formation and gamma-ray emission in star-forming galaxies. Moreover, direct observations of SNRs have also shown that the locations of gamma ray emissions are compatible with regions heated by shocks. In this case, detections of SNRs at $\mathrm{GeV}$ energies are complementary to the detections at higher energy by ACT. Particles accelerated in shocks cool down to lower energies and only young SNRs can be detected at TeV energies. Middle-aged SNRs on the other hand are still visible to the LAT. In general, it is believed that emission of gamma-rays in SNRs can be generated by leptonic processes, such as synchrotron, inverse Compton or bremsstrahlung scattering, or from hadronic cascades, such as photo-pion production. As illustrative example, Fig. 1 shows the young SNR Cassiopeia A detected by Fermi, MAGIC, and VERITAS. Both leptonic models and hadronic models can describe the data with accurate precision, and no "smoking gun" has been found yet, but it is clear that understanding the relative importance of leptonic and hadronic processes requires combined multiwavelength observations.
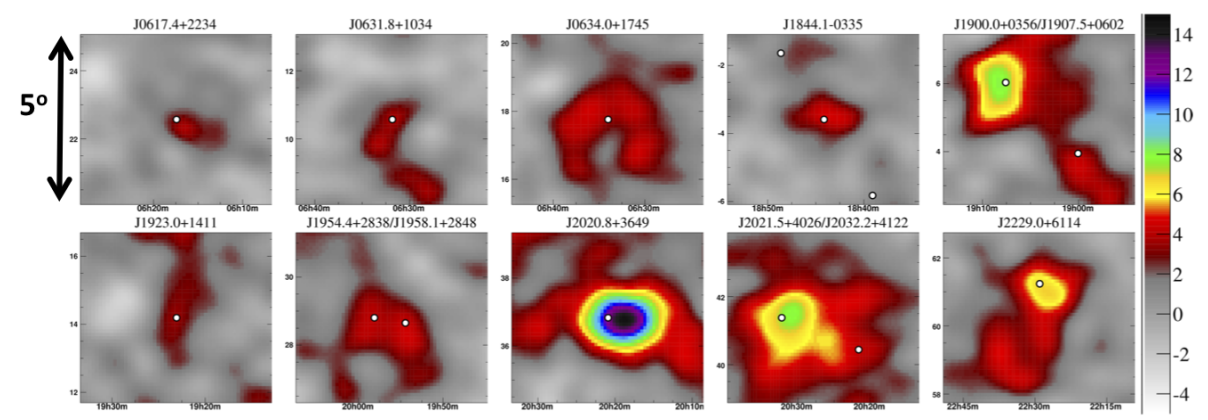

Figure 2: Milagro observation of multi-TeV emission from Galactic sources associated with Fermi Galactic sources. The colors indicate the statistical significance in standard deviations.

Finally, a nice example of synergy between the experiments comes from the Milagro collaboration[9], which reports the detection of high-significance sources associated with Fermi pulsars (Fig. 2). The emission from gamma-ray pulsars rolls off steeply at $\mathrm{GeV}$ energies due to the cooling of particles in the high magnetic field in the outer gap of the neutron star. On the other hand, pulsars are superb accelerators of particles, which, escaping from the central region, interact with surrounding nebula, emitting radiation at $\mathrm{TeV}$ energies. The strong evidence for multi- $\mathrm{TeV}$ emission associated with Galactic LAT sources favors the interpretation that the typical Galactic multi-TeV source is indeed a pulsar wind nebula (PWN) associated with a $\mathrm{MeV} / \mathrm{GeV}$ pulsar.

\section{Cosmic Rays}

New results have been presented at this conference covering, approximately, the entire spectrum of cosmic-ray particles detected at Earth, from above the geomagnetic cut-off (few $\mathrm{GeV}$, for electrons), to and above the GZK suppression, at approximately, $4 \times 10^{19} \mathrm{eV}$.

Results from the PAMELA satellite[10] include the antiproton flux and the antiproton-toproton flux ratio (in the range $0.06 \mathrm{GeV}-180 \mathrm{GeV}$ ), the preliminary electron flux up to $200 \mathrm{GeV}$, 
and the new measurement of positrons-to-all-electrons ratio[11] (Fig. 3, right). Preliminary results on proton and Helium up to $1 \mathrm{TeV}$ were also presented. They reported a change of slope in both of the spectra at energy (rigidity) of $300 \mathrm{GeV} / \mathrm{n}(230 \mathrm{GV})$ for protons and $100 \mathrm{GeV} / \mathrm{n}(260 \mathrm{GV})$ for Helium.
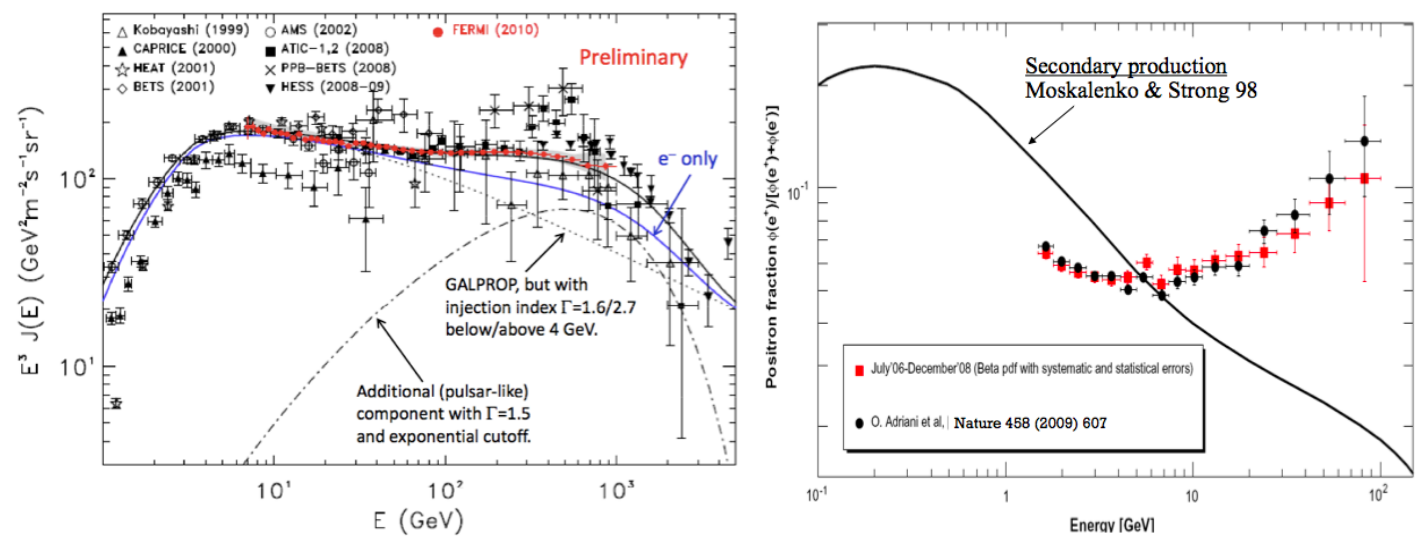

Figure 3: Left: Fermi electron-positron flux: an additional component of electrons and positrons (pulsarlike) would introduce a hardening of the spectrum at $\approx 50 \mathrm{GeV}$ followed by a cut-off, representing very well both Fermi and H.E.S.S. measurements. The same additional component would also naturally explain the excess observed by PAMELA in the positrons-to-all-electrons flux ratio (right). Different interpretations, including DM scenarios, have been explored by several authors (see for example[12]).

The Fermi collaboration[5] reported a new measurement of the CR electron-plus-positron spectrum[13], with increased statistics that extend the spectrum down to $7 \mathrm{GeV}$ and up to $1 \mathrm{TeV}$ (Fig. 3, left). In addition to this, a comprehensive analysis of spatial anisotropies has also been presented[14]. In this respect, an asymmetry oriented in the direction of a nearby CR source would favor an "astrophysical" origin for the increase of the positron ratio observed by PAMELA and would naturally explain the extra component needed above the conventional model to explain the excess at $\approx 50 \mathrm{GeV}$ in the Fermi spectrum. A contribution from DM particles annihilating in the Milky Way halo, on the other hand, would result in a low degree of anisotropy ${ }^{3}$. No anisotropy has been found, but the scenario where both the Fermi and PAMELA "excesses" are due to the presence of nearby sources is not excluded by the $68 \%$ CL upper limits reported by Johnson in his talk.

At higher energies (between 5 and $250 \mathrm{TeV}$ ), the spectrum of the light $\mathrm{CR}$ component ( $\mathrm{p}+\mathrm{He}$ ) has been presented by the ARGO-YBJ collaboration[15]. The proton spectrum is flatter than in the lower energy region, in agreement with CREAM data, and confirming the change of slope observed by PAMELA at lower energies.

Even more puzzling, regarding the directions of incoming protons in both ARGO-YBJ and Milagro data (see Fig. 4), two localized regions of CR excess are significantly detected in both the celestial maps (at more than $10 \sigma$ ). Different ideas have been proposed to explain this intriguing observation, manifesting in particular the need for reviewing the origins of Galactic CRs and propagation including the effects of the interaction of the solar system with the interstellar medium (in

\footnotetext{
${ }^{3}$ A clumpy DM structure of sub-halos would introduce some anisotropy also in this scenario
} 

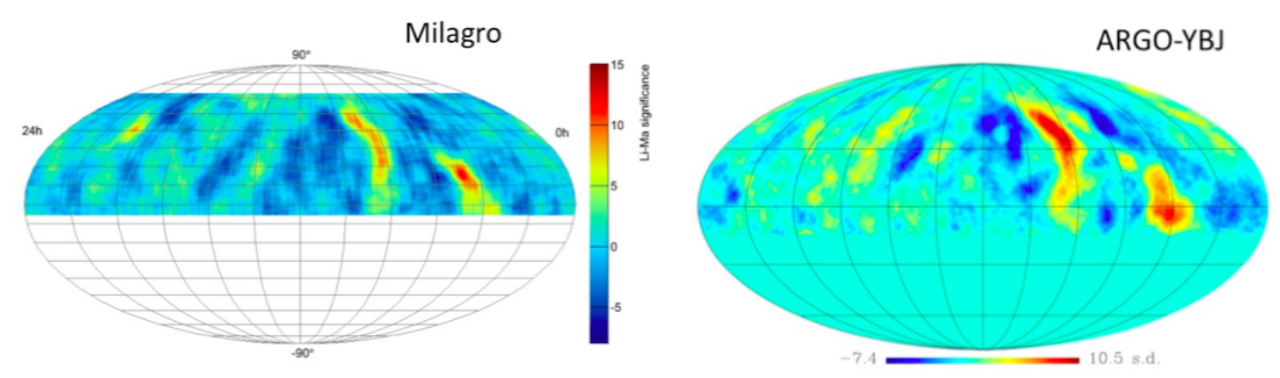

Figure 4: Anisotropy map in celestial coordinate from Milagro and ARGO-YBJ. Medium scale anisotropies ("Hot Spots") are clearly visible in both of the maps at high significance (>10 s.d.).

the heliosphere)[16].

At very high energies $\left(5 \times 10^{19} \mathrm{eV}\right)$ protons can travel only $\approx 10 \mathrm{Mpc}$ before producing pions due to interactions with the cosmic microwave background (the GZK effect[17, 18]). On the other hand, strong evidence indicates that CRs above $10^{17} \mathrm{eV}$ have an extragalactic origin: this results in a abrupt steepening of the spectrum above the energy of the cutoff. HiRes[19], Telescope Array (TA)[20] and Pierre Auger Observatory (PAO) [21] reported results for this energy range. First, the GZK suppression is evident in all the experiments (at 5.3, 3.5 and and $20 \sigma$ level, respectively). Even more interesting, the correlation of the events with energy greater than $6 \times 10^{19} \mathrm{eV}$ with AGN claimed in 2007 by the PAO collaboration[22], dropped from a degree of $69 \%$ to $38 \%$ (at 99\% CL). The latest results are compatible ( 0.3 by-chance probability) with an isotropic distribution.

Finally, I also have found very interesting the work reported by Thomson in his talk[20] on the careful validation of Monte Carlo codes used to simulate CR showers developing in the atmosphere (CORSIKA/QGSJet). This is a key study to constrain the uncertainties on energy estimation, needed to address the problem of the composition of CRs at the Earth. For example, the mean of the distribution of the maximum of the hadronic shower $\left(\left\langle X_{\max }\right\rangle\right)$ is a standard parameter to describe the development of a shower in the atmosphere, and the estimation of the energy of the incoming particle relies on it. Calibration of this quantity is based on MC simulations. First results showing the composition of CRs at the ground reported by HiRes and PAO are in disagreement. This disagreement certainly could be due to the different sky the two observatories see, but also could be due to systematic bias in the determination of the energy scale. In this context, a link with the LHC is clear: measurements from $p p$ collisions help to understand and validate MC codes, and reduce the uncertainties related to the difficult task of simulating hadronic interactions.

\section{Multimessenger}

In the final part of my talk I remarked on a couple of interesting results and progresses from neutrino astrophysics and gravitational waves detectors. For the first, the take-home plot that summarizes the current results is in Fig. 5. At lower energies the measurements (IceCube[23], Antares[24]) are related to the atmospheric neutrinos $\left(v_{\mu}\right)$ which is the dominant contribution at low energies. At higher energies, the contribution from atmospheric neutrinos rolls off and it is believed that celestial neutrinos become the dominant contribution. Upper limits from Antares, Auger and IceCube are reported (all normalized to the rates of the three flavors). Dashed and dotted lines 
indicate the expected fluxes from different sources of celestial neutrinos (GRB and AGN), while at high energies the expected contribution from GZK neutrinos is also shown (ESS model) ${ }^{4}$

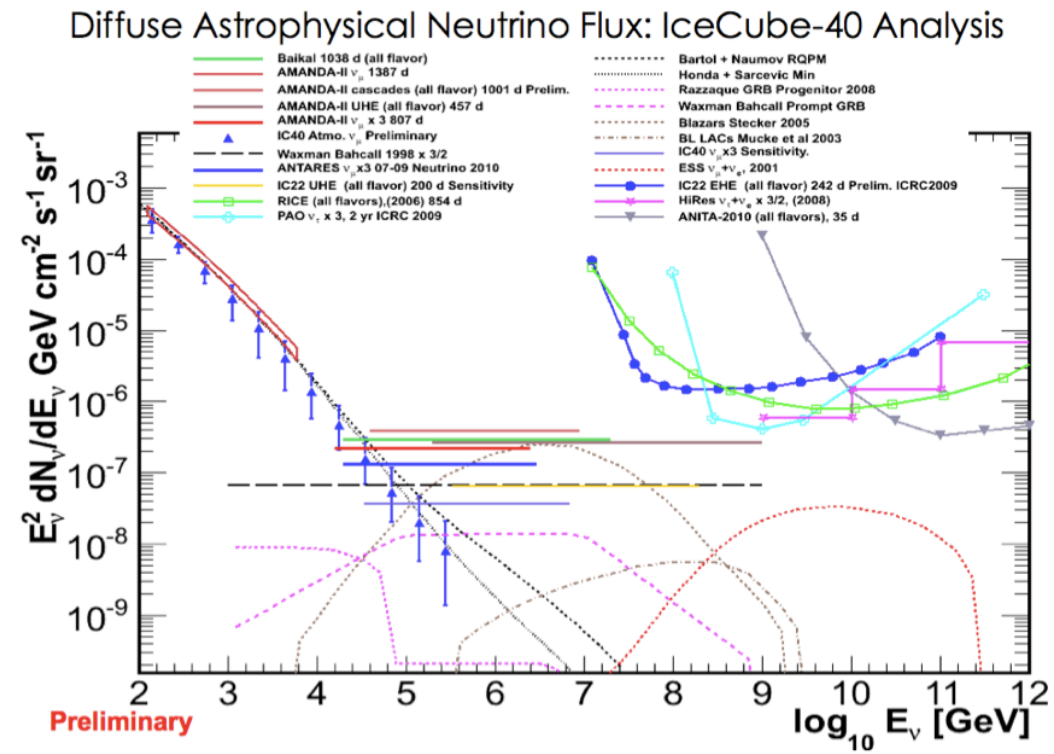

Figure 5: At low energy: measurements of atmospheric neutrino flux. High energies: upper limit on the flux of celestial neutrinos presented at ICHEP 2010 from Antares[24], Auger[21] and IceCube[23]. Fluxes are normalized to the rates of the three flavors.

Gravitational wave GW interferometers[25] have reached the so-called "Enhanced Detector Phase" increasing their sensitivity by a factor of 10 with respect to the first generation. Still a detection at current sensitivity limits is unlikely, but interesting upper limits have been reported. With data combined from various interferometers, new limits on the event rate of coalescing binaries have been reported $\left(8.7 \times 10^{-3} y r^{-1}\right.$ for binary neutron stars, $2.2 \times 10^{-3} y r^{-1}$ for black hole neutron star systems, and $4.4 \times 10^{-4} y r^{-1}$ for binary black hole systems)..$^{5}$ The "spin down limit" is reached for three pulsars (i.e., pulsars cannot emit only GW or they would have been detected ${ }^{6}$ ). A new upper limit on the stochastic gravitational-wave background also has been set (in terms of density divided by the critical density: $\Omega_{0}<6.9 \times 10^{-6}$ ) and lower limits on the distances of GRBs detected by Swift have also been presented. In this context I found particularly interesting the possible synergy between GW detectors, neutrino telescopes and X-ray and gamma-ray telescopes, such as Fermi or Swift. Localizations of the sources from Fermi or Swift could improve the sensitivity to GW signals due to the narrowing (in space and time) of the search windows. To this end, real time data analysis and rapid follow-ups have been already tested and look very promising, especially in the prospect of the new generation of interferometers "Advanced Detector" phase (2011-2020).

\footnotetext{
${ }^{4}$ When the protons collide with the microwave background photons, mesons are created and high-energy neutrinos are produced in their subsequent decays.

${ }^{5}$ The rates are in units of number per year assuming a binary coalescence rate proportional to $10^{10}$ times the blue solar luminosity.

${ }^{6}$ We know that a certain fraction of their spin down luminosity goes into gamma rays, X-rays, and other wavelengths.
} 


\section{Conclusions}

It is a Golden age for Astroparticle physics, with a big boost given by the Fermi telescope operating as a path finder providing a huge collection of gamma-ray sources. In this context the synergy between experiments is important. From my perspective, development of technologies that will continuously increase the sensitivity of the detectors is only one key for expanding our current knowledge. It is of great importance to match this with the development of systems to quickly provide alerts in cases of something interesting happening in the sky. The current experience shows that fast communications (using GCN, ATel or ad-hoc socket connections) and collaborative efforts between experiments are very important for obtaining a broad picture of the transient phenomena, without which complete understanding cannot be achieved. Collaborative efforts have indeed shown the payoff. Gamma-Ray Bursts detected by gamma-ray telescopes are then followed by X-Ray, optical and radio telescopes until their emission has, after weeks or even months, completely faded. We are now starting to think about ways to target GRBs also with neutrino telescopes and GW interferometers, and particle acceleration in Super Nova Remnants in our and in other galaxies has been simultaneously studied at all wavelengths, to discover the link between hot shocked gas and particles being accelerated. Concerning Dark Matter searches, the tight connection with particle physics extends beyond the Standard Model suggesting that a discovery at LHC would represent a revolution for Astroparticle physics as well.

\section{References}

[1] Larson, D., et al. 2011, ApJS, 192, 16

[2] Kos, M., for the CDMSII collaboration, ICHEP 2010 (these proceedings).

[3] Hall, C., for the LUX collaboration, ICHEP 2010 (these proceedings).

[4] Lin, Shin-ted, for the CJPL collaboration, ICHEP 2010 (these proceedings).

[5] Johnson, R., for the Fermi LAT collaboration, ICHEP 2010 (these proceedings).

[6] Glicenstein, J. F., for the H.E.S.S. collaboration, ICHEP 2010 (these proceedings).

[7] Abdo, A. A., et al. 2010, ApJS, 188, 405

[8] Hanna, D., for the VERITAS collaboration, ICHEP 2010 (these proceedings).

[9] Westerhoff, S., for the Milagro collaboration, ICHEP 2010 (these proceedings).

[10] Adriani, O., for the PAMELA collaboration, ICHEP 2010 (these proceedings).

[11] Adriani, O., et al. 2009, Nature, 458, 607

[12] Grasso, D., et al. 2009, Astroparticle Physics, 32, 140
[13] Ackermann, M., et al. 2010, Phys. Rev. D, 82, 092004

[14] Ackermann, M., et al. 2010, Phys. Rev. D, 82, 092003.

[15] Marsella, G., for the ARGO-YBJ collaboration, ICHEP 2010 (these proceedings).

[16] Abdo, A. A., et al., 2008, Phys. Rev. Lett., 101, 221101.

[17] Greisen, K. 1966, Phys. Rev. Lett.16, 748

[18] Zatsepin, G. T., \& Kuz'min, V. A. 1966, Soviet Journal of Experimental and Theoretical Physics Letters, 4, 78

[19] Sokolsky, P., for the HiRes collaboration, ICHEP 2010 (these proceedings).

[20] Thomson, G., for the Telescope Array (TA) collaboration, ICHEP 2010 (these proceedings).

[21] Kampert, K., for the Pierre Auger Observatory (PAO) collaboration, ICHEP 2010 (these proceedings).

[22] The Pierre Auger Collaboration, et al. 2007, Science, 318,938

[23] Finley, C., for the IceCube collaboration, ICHEP 2010 (these proceedings).

[24] Spurio, M. for the Antares collaboration, ICHEP 2010 (these proceedings).

[25] Cavalier, F. for the LIGO Scientific Collaboration and Virgo collaboration, ICHEP 2010 (these proceedings) 